\title{
A Study Investigating the Use of 3D Computer Animations of Trigonometric Functions to Enhance Spatial Perception Ability
}

\author{
Ting-Sheng Weng, Meng-Hui Hsu, and Der-Ching Yang
}

\begin{abstract}
This study developed teaching materials of life application simulation animations, and introduced the concept of trigonometric functions into actual stories to create animations. This study took the famous ancient Chinese mathematical measurement book, Hai Dao Suan Jing for example, and created 3D simulation animations, auxiliary lines, and graphics. It is expected to help students to understand the allusions and mathematical formulas of Hai Dao Suan Jing, and learn to use their height, change of location, and change of related projection to measure the height and distance of objects on the ground. By watching 3D simulation animations, students can better understand the mathematics questions through dynamic visualization, learn the principles of surveying, and learn the spatial conception of trigonometric functions. The use of teaching with computer animations to attract students' curiosity regarding the application of mathematics to life can improve their imagination about surveying and simulation of spatial concepts.
\end{abstract}

Index Terms-Hai Dao Suan Jing, surveying, spatial ability, visual animations, computer education.

\section{INTRODUCTION}

Spatial ability is extremely beneficial to the development of intellectual potential and logic training. Therefore, the training of spatial ability is not only the focus of teaching a mathematics course, but is also an indispensable critical ability for the development and use of 3D animation games.

In traditional teaching of mathematics and physics, written materials or physical model sets are usually used to teach students, thus, there is a lack of application of exposure to three-dimensional visual spaces.

To the digital native generation [1], [2], the use of a computer and the internet is an inevitable trend. Compared with the previous generation, students who are born after the 1980s particularly have more opportunities to be exposed to all kinds of digital information technologies. Students of the new generation use instant messaging software and social media to obtain social messages and feedback. They use abundant graphic interfaces in combination with the windows

\footnotetext{
Manuscript received June 6, 2015; revised September 23, 2015.

Ting-Sheng Weng is with the Department of Business Administration, National Chiayi University, Taiwan (e-mail: politeweng@mail.ncyu.edu.tw).

Meng-Hui Hsu is with the Department of Mechanical Engineering, National Cheng-Kung University, Taiwan (e-mail: mhhsu@mail.ksu.edu.tw).

Der-Ching Yang is with the Institute of Mathematics and Science Education, National Chiayi University, Taiwan (e-mail: dcyang@mail.ncyu.edu.tw).
}

of operation and information searches that they use. As a result, the learners of the digital native generation are more accustomed to graphic stimulation, parallel processing, and immediate response [3]. Therefore, they have increasing opportunities to be exposed to three-dimensional visual space. Flat space is two-dimensional space, and is usually reflected by written materials or on a screen. As it progresses to stereoscopic space, namely, three-dimensional space, different visual effects will be generated in the reflection of written materials or a screen, and further lead to visual bias caused by different angles. Therefore, in order to further investigate the differences in visual effects caused by space combinations, as well as their importance to mathematics education, animations are used to reflect the effect. The learning environment buildings of new generations should be featured by instant learning, interactions, learner control, multimedia, and reflection [4]. Therefore, animations possess a part of the functions that enable students' learning to become more diversified and autonomous.

\section{Motivation AND PURPOSE}

In recent years, academia has aggressively promoted the teaching objective of multiple intelligence learning. For example, the content of the mathematics course field includes the concepts of graphics and spatial ability in multiple intelligences. However, because there exists an urban-rural gap in teaching environments, many school teachers' instruction of 3D graphics and geometry are restricted to the visual effects of 2D space on a whiteboard, which prevents students from re-developing graphic memories and thinking, and leads to a failure to accurately identify the distance between objects and selves, and the size of objects, as well as a failure to regroup the objects they see. Therefore, this study developed the teaching aids of animations for students to use, in order to improve their ability of spatial conception, ability to learn the concepts of mathematical trigonometric functions, and achieve a more intuitive imagery connection during accurate and rapid re-reversal of images.

\section{LITERATURE REVIEW}

Literature [5] divided individuals' spatial knowledge into two categories: meanings and understandings, which refers to individuals' understanding of geometrical concepts; and abilities presses, which refers to individuals' ability to imagine space and matter. Similar concepts were proposed in literature [6]. Spatial knowledge is divided into two 
categories: geometric thinking and spatial thinking: spatial visualization and spatial orientation [7]. Spatial ability has a profound influence on understanding scientific concepts [8]. Teachers tend to use the teaching materials and teaching aids provided by publishing houses to engage in teaching. Such teaching lacks an understanding of students' cognitive development, ignores whether students can thoroughly grasp the characterization phenomena of teaching materials, and further discovers that visualization teaching aids, such as dynamic graphics and 3D models, are indeed beneficial to students' development of accurate imagery and understanding of abstract scientific concepts. Therefore, enhancing spatial ability is an important direction of scientific education.

Spatial visualization ability refers to individuals' ability to play, rotate, twist, or reverse images to reflect the stimulation effect in the mind. Spatial orientation ability refers to individuals' understanding of a series of responses caused by stimulation, as well as the relationship with the original unstimulated status. After the directions of reflected spatial conception are changed, individuals' ability will not be undermined.

Literature [9] used innovative virtual mathematics games to increase students' interest in mathematical learning, and improved their learning effectiveness by developing a mathematical learning computer game system. They found that, the use of an interesting mathematical animation game can increase students' desire to learn and improve their learning effectiveness. In addition, mathematical animation games can be comprehensively applied to other courses, such as statistics or calculus.

Regarding the actual application of spatial ability, such as engineering mechanics, after the use of animation simulation teaching, 58\% 88\% of students indicated positive learning experiences, while $36 \%$ 85\% suggested that they benefited greatly from such teaching [10]. When the level of complexity of images is higher (i.e. various cubes and compositions, especially irregular shapes or concave cubes), the use of a 3D coloration model to engage in animation teaching is more beneficial to the development of the graphic vision of objects [11].

Literature [12] investigated the application of calculus to the induction of the calculation of the movement trajectory of a parabolic sphere. If animations are used to reflect the change in directions of tangential velocity of every point on the movement trajectory of a sphere, learners can understand the physics meaning of calculus reflected in geometric application, as well as the relationship between the tangent slope of a trajectory and trigonometric functions. Moreover, they indicated that, the use of animations can improve students' learning interest and effectiveness in their learning of curves of functions, as well as their derivatives.

\section{A. Importance of Spatial Visualization Ability}

Spatial ability is based on general cognitive ability, and is the ability to generate imagery caused by external stimulation to further control imagery and solve problems [13]. In other words, spatial ability refers to individuals' thinking ability to accurately observe and identify objects, and memorize them in the mind, as based on the images visually absorbed, to further use imagination to arbitrarily move, rotate, or reverse objects in 2D and 3D spaces to reflect the re-identification, retention, and memory of images. Most students in Taiwan are deficient of such cognition and ability to generate internal imagery, and thus, encounter difficulties in object identification and space combinations of graphics. If such learning resources can be integrated by a Computer education environment, students' space construction ability can be improved.

Literature [14] investigated the spatial ability to form imagery (namely, the ability to manipulate 2D and 3D spaces), as well as the influence of visual characterization (symbols and image characterization) on imagery formation, and its application to scientific education. To students, their ability to learn space in class is learned from planes, and such learning effectiveness is limited. The actual example of spatial ability is similar to the size of an object perceived by people in a general environment, which can be estimated after judgment by the brain. In fact, they are life abilities. Therefore, enhancing students' spatial ability is extremely beneficial to the judgment of actual objects.

\section{RESEARCH APPROACH}

The main purpose of this study is to produce animations for social application of spatial ability, as well as to introduce animations with educational meanings into the learning process of spatial perception and spatial ability.

\section{A. Research Tools}

This study designed the animation teaching materials of Hai Dao Suan Jing, which contain applications of trigonometric functions, as based on the content of the book Hai Dao Suan Jing (p. 339 of Unit 4) in the textbook of new mathematics classroom for senior high school (published by Chien Hong Publishing House in 2007). This study also used software, such as Flash CS4 and Google SketchUp, to design animation teaching materials to teach students to understand the specific concepts of the use of Displacement to measure the height and distance of an object on vacant ground. This study designed a question of animation teaching materials regarding the use of displacement to measure the height and distance of an object on the ground, as follows:

"In a certain war, the task of the scout is to observe the guard standing on the top of lighthouse on the island. In order to precisely measure his position, it is necessary to calculate the distance between the closest point on the land of our army and the lighthouse, as well as the height from top of the lighthouse to sea level."

Therefore, as shown in Fig. 1, the lighthouse is located at point $\mathrm{D}$, and the spotlight on the top of lighthouse is located at point E. The spotlight projects the scout located at different positions (points $A_{1}$ and $A_{2}$ ) on the same straight line onto points $C_{1}$ and $C_{2}$. The scout uses the distance between his position (point $A_{1}$ ), the lighthouse, and the height of the lighthouse for measurement. If the height of the scout is $t$ and he stands at different positions (points $A_{1}$ and $A_{2}$ ) at the distance of $d_{1}$, his head is located at points $B_{1}$ and $B_{2}$, 
respectively, and his height projected by spotlight is $s_{1}$ and $s_{2}$, respectively.

As shown in Fig. 1, this study designed a question of animation teaching material, which is also the use of displacement to measure the height and distance of an object drawn on a whiteboard by senior high school teachers. Because the learning of spatial concepts to train the stereoscopic perception ability of 3D visual effect is beneficial to the conversion of planar graphics into accurate stereoscopic shapes by the brain, this study used software, such as Flash CS4 and Google Sketch Up 8, to develop reversible 3D animation concerning the teaching content, where trigonometric functions and displacement are used to measure the height and distance of an object, as shown in Fig. 2 , and assist students in improving their spatial perception ability.

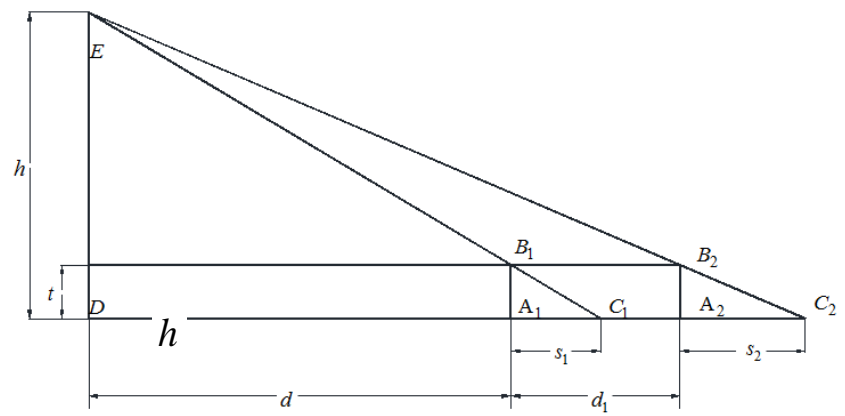

Fig. 1. The Scout's measurement of distance $(d)$ and height $(h)$ of the lighthouse.

As shown in Fig. 1, two rectangular triangles, $\Delta C_{1} A_{1} B_{1}$ and $\triangle C_{1} D E$, are similar. Two rectangular triangles, $\Delta C_{2} A_{2} B_{2}$ and $\Delta C_{2} D E$, are similar. In other words, $\triangle C_{1} A_{1} B \sim \Delta C_{1} D E$ and $\Delta C_{2} A_{2} B_{2} \sim \Delta C_{2} D E$. According to the tangent function and proportional side lengths in similar triangles, the following formulas can be obtained:

$$
\begin{gathered}
\tan \left(\angle E C_{1} D\right)=\frac{t}{h}=\frac{s_{1}}{s_{1}+d} \\
\tan \left(\angle E C_{2} D\right)=\frac{t}{h}=\frac{s_{2}}{s_{2}+d_{1}+d}
\end{gathered}
$$

Eqs. (1) and (2) can be used to calculate the distance between point $A_{1}$ and the lighthouse, and the height of the lighthouse as:

$$
\begin{gathered}
d=\frac{s_{1} d_{1}}{s_{2}-s_{1}} \\
h=\left(1+\frac{d_{1}}{s_{2}-s_{1}}\right) t
\end{gathered}
$$

If the scout at the height of $t=180 \mathrm{~cm}$ stands at different positions (points $A_{1}$ and $A_{2}$ ) at the distance of $d_{1}=600 \mathrm{~cm}$, the height projected by the spotlight is $s_{1}=300 \mathrm{~cm}$ and $s_{2}=420 \mathrm{~cm}$, respectively. According to Eqs. (3) and (4), the distance $d$ between point $A_{1}$ and the lighthouse, and the height of lighthouse $h$ can be obtained:

$$
\begin{gathered}
d=\frac{s_{1} d_{1}}{s_{2}-s_{1}}=\frac{300 \times 600}{420-300}=1500 \mathrm{~cm} \\
h=\left(1+\frac{d_{1}}{s_{2}-s_{1}}\right) t=\left(1+\frac{600}{420-300}\right) 180=1080 \mathrm{~cm}
\end{gathered}
$$

\section{B. Animation Design}

In order to enable students to learn to use trigonometric functions and displacement to measure the height $(h)$ and distance $(d)$ of an object on the ground, this study designed integrated 3D animations, as shown in Fig. 2, in Flash CS4 and Google SketchUp. The context in this animation design is, as follows:

"The scout finds that he cannot directly measure the height of the lighthouse because there is an ocean between him and it, and he may be discovered by the enemy. Therefore, he has to use his height and the projection of his height at different position, as well as trigonometric functions and displacement, in order to measure the height $(h)$ and distance $(d)$ of the lighthouse."

Such a design enables teachers to explain to students, as well as to let them watch animations. This approach is also beneficial to students' conversion of 2D graphics to accurate 3D stereoscopic shapes. Moreover, students can review and watch animations after class to increase their understanding.
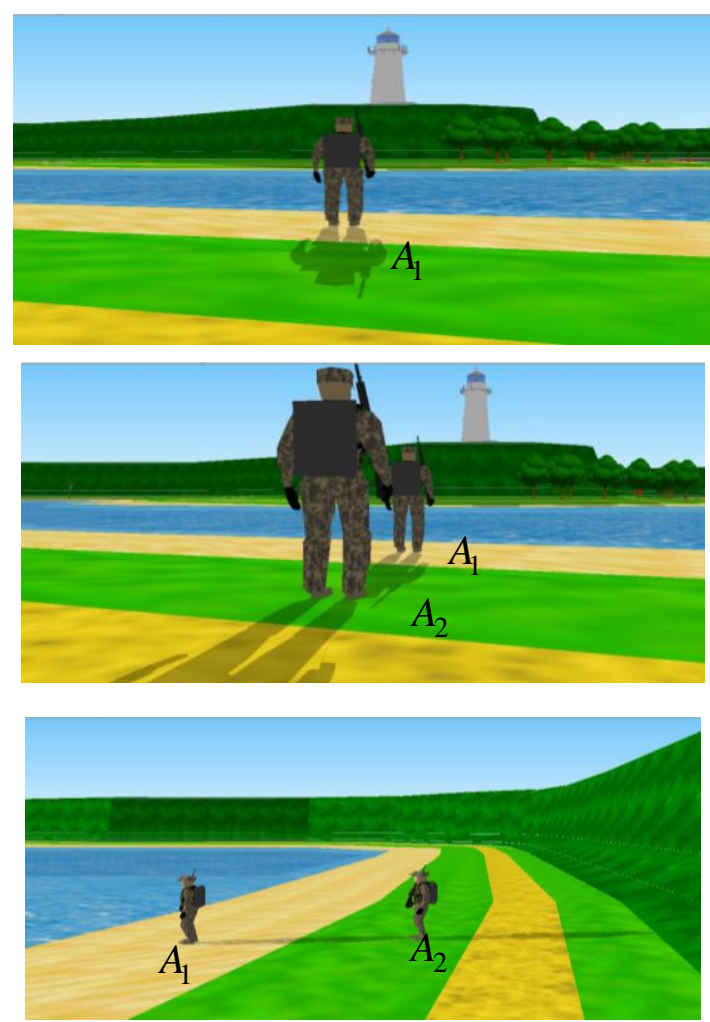


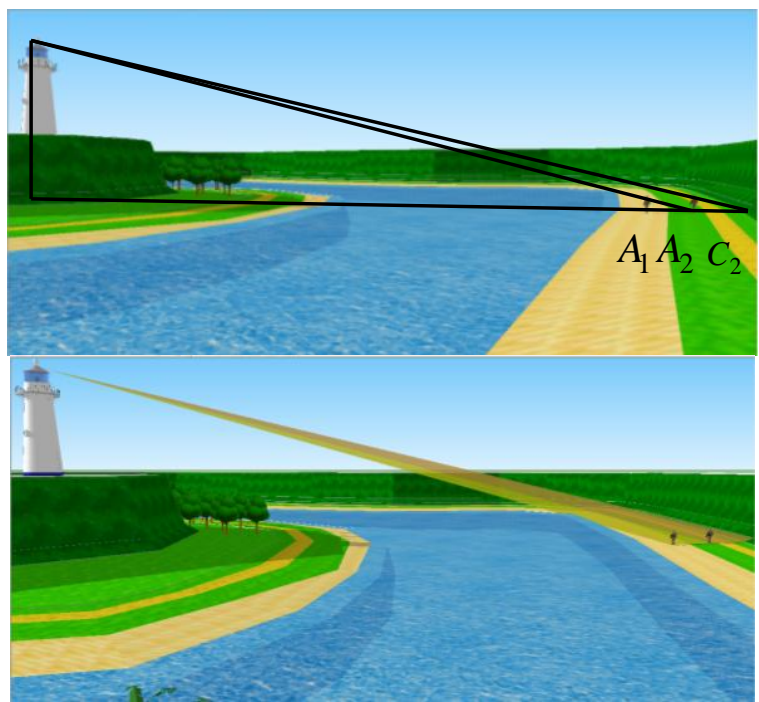

Fig. 2. Animation of the use of trigonometric functions and displacement to measure the height and distance of an object.

\section{CONCLUSION}

As 3D spatial perception ability enables people to understand their surrounding space, good perception ability enables people to clearly understand the relative positions and relevant distances of objects in their current environment. 3D surrounding space allows learners to better understand the relative spatial objects of all people, things, and matters around them. The use of trigonometric functions and the introduction of mathematical formulas of proportional side lengths in similar triangles into SD space enables learners to clearly perceive the meanings and concepts represented by trigonometry in real life, and thus, improve their learning effectiveness.

The 3D reflection of traditional narrative stories can enable receivers of stories to be directly exposed to images and decrease the error caused by imaginary space, which makes story receivers better perceive story content, provides them with deeper impressions in the mind, and enables them to easily memorize stories for a longer period of time. This study developed the 3D simulation animation story, where trigonometric functions and displacement are used to measure the height and distance of an object to enable students to understand the allusions and mathematical formulas of $\mathrm{Hai}$ Dao Suan Jing, and learn to use their own height, change in positions, and change in relevant projection to measure the height and distance of an object on the ground. Such animation can be used as life application education teaching materials, mathematical learning teaching materials, and teaching aids of computer education for developing spatial concepts.

\section{ACKNOWLEDGEMENTS}

The author appreciates the comments of the review committee. This project was supported by the Ministry of Science and Technology No. MOST103-2511-S-415-002.

\section{REFERENCES}

[1] M. Prensky, "Digital natives, digital immigrants," On the Horizon, vol. 9, no. 5, pp. 1-6, 2001.
[2] H. F. Gao, Learning and Teaching of Digital Natives, Scientific and Technological Development and Educational Reform, Taipei: Higher Education, pp. 1-18, 2013.

[3] C. F. Chen and C. C. Ji, Learning and Teaching of Digital Natives, Learning Tools and Learning Environment of Digital Native Generation, Taipei: Higher Education, pp. 21-39, 2013.

[4] A. Collins and R. Halverson, Rethinking Education in the Age of Technology: The Digital Revolution and Schooling in America, New York: Teachers College Press, 2009

[5] A. J. Bishop, "Space and geometry," in R. Lesh and M. Landau Eds., Acquisition of Mathematics Concepts and Processes, New York: Academic Press, pp.125-203, 1983.

[6] D. H. Clements and M. T. Bttista, "Geometry and spatial reasoning," Handbook of Research on Mathematics Teaching and Learning, New York: Macmillan, 1992, pp. 420-464.

[7] S. L. Huang and S. C. Ghou, "The analysis about interaction process and solving problem strategies of different scaffolding supportive styles in the Internet learning environment," NSC93-2413-H-260-005, 2004.

[8] H. H. Lin, C. T. Hsiung, and S. H. Lin, "CISC instructional strategy on the spatial ability of junior high students," Bulletin of Educational Psychology, vol. 37, no. 4, pp. 393-409, 2006.

[9] Y. G. Yang and L. Li, International Journal of Computer Theory and Engineering, vol. 4, no. 4, 2012.

[10] N. Fang, "Using computer simulation and animations to improve student learning of engineering dynamics, procedia - social and behavioral sciences," in Proc. International Conference on Teaching and Learning in Higher Education, vol. 56, pp. 504-512, 2012.

[11] C. F. Wu and M. C. Chiang, "Effectiveness of applying 2D static depictions and 3D animations to orthographic views learning in graphical course," Computers and Education, vol. 63, pp. 28-42, 2013.

[12] T. S. Weng, M. H. Hsu, D. C. Yang, and J. Y. Lin, "Verification, evaluation, and design of applying calculus to construct 3 danimationss of kinematic and force states," NSC101-2511-S415-006, 2012.

[13] H. L. Jeng and S. Y. Chen, "Model exploration and validation of the standardized spatial ability test," Journal of Educational Research and Development, vol. 3, no. 4, pp. 181-216, 2007.

[14] C. J. Liu, "The study on the effects of spatial ability and visual representation on forming mental image in learning science," NSC96-2511-S017-002-MY3, 2009.

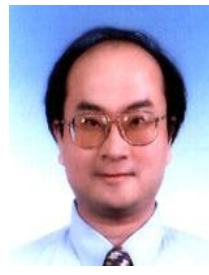

Ting Sheng Weng is an assistant professor of the Department of Business Administration at National Chiayi University, Taiwan. He received his $\mathrm{Ph}$. D. in Division of Management and Information Science, Graduate School of Advanced Technology from Kinki University, Japan in 2000. He had served as a visiting professor at Meiji University in Japan from 2006 through 2007. His major research interests include science technology and management, e-learning, information education, technology education, MIS, and image processing.

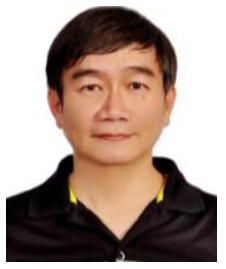

Meng-Hui Hsu took his M. S. and Ph.D. from the Department of Mechanical Engineering, Nationa Cheng-Kung University, Taiwan, 1990 and 1996, respectively. Now Dr. Hsu is an associate professor of Mechanical Engineering Department at Kun-Shan University in Taiwan. His research interests include mechanism and machine design, theory of creative design, and servo control systems.

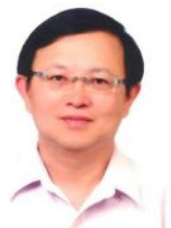

Der-Ching Yang is a professor of the Graduate Institute of Mathematics and Science Education at National Chiayi University. Dr. Yang received his $\mathrm{PhD}$ in mathematics Education from University of Missouri-Columbia in 1995. His research focuses on number sense, mathematics teachingg and learning, technology \& mathematics teaching and learning, curriculum study. 